\title{
Analysis of the load capacity of the bolt joints of collecting electrodes with the rapper rod
}

\author{
Andrzej Nowak ${ }^{1, *}$, and Mirostaw Kurz ${ }^{2}$ \\ ${ }^{1}$ University of Bielsko-Biala, Willowa 2, 43-309 Bielsko-Biała, Poland \\ ${ }^{2}$ Dedusting Installation Division, Rafako S.A., Górnośląska 3A, 42-200 Pszczyna, Poland
}

\begin{abstract}
This article presents an analysis of the load capacity of the bolt joints of collecting electrodes with the rapper rod, made in HUCK technology. A discrete model of the system of electrodes was worked out for the analysis of stresses in bolt joints, the validation of which was made on the basis of measurement data obtained on the test bench. Two variants of joining the plates of electrodes with the rapper rod were taken into account in calculations - the first one with the correct geometry in the rapper rod, the other one with an eccentric displacement of holes in the flat bars of the rapper rod. In both variants the modelled system was loaded with the impulse force corresponding to the impact of the hammer against the anvil, with the run obtained from measurements on the test bench. These models and the related calculations were performed in the ABAQUS environment. As a result of the analyses performed, recommendations were worked out intended to eliminate cases of the destruction of bolt joints closest to the anvil of the rapper rod.
\end{abstract}

\section{Introduction}

This article presents an analysis of the load capacity of the bolt joints of collecting electrodes with the rapper rod, made in HUCK technology. In the structure tested, the collecting electrodes of an electrostatic precipitator are periodically cleaned by inducing violent vibrations in them - the acceleration values definitely exceed $1000 \mathrm{~ms}^{-2}(1)$. These vibrations are caused by the impulse forces of drop-weight hammer impacts in the anvils of rapper rods [2]. Transfer of vibrations from the rapper rods to collecting electrodes is carried out by the bolt joints analysed in the study. The quality and stability of these joints is one of the important factors which indirectly affect the efficiency of cleaning exhaust gases from solid particles in electrostatic precipitators.

\section{Models of the system}

The models shown further on, and the related calculations were performed in the ABAQUS environment. Parts of the system were modelled using deformable discrete components described by the finite element method (FEM) [3], [4] - electrodes were

\footnotetext{
* Corresponding author: a.nowak@ ath.bielsko.pl
} 
modelled with coat components of the I order (shell, S4R), and the remaining parts of the system with continuous components also of the I order (solid, C3D8R) [5]. The upper, articulated suspension of the electrodes in the beam was performed as a mobile, rotary joint of hinge type. In the bolt joints friction was taken into account - accepted coefficient of friction $\mu=0.4$ - according to the idea of stressed or rivet joints, where the load capacity of the joint is obtained by the effect of friction induced by compression, and not by shearing and pressure as in conventional joints. Validation of models was based on the measurement data obtained on the test bench, as in earlier works of the co-authors [1], [6].

\subsection{FEM model of the collecting electrodes}

Two discrete models of the system of 9 collecting electrodes were used in the analysis of stresses in bolt joints consisting of 2 reinforced electrodes and 7 standard electrodes of the type SIGMA VI, length $16400 \mathrm{~mm}$, made of sheet with a thickness of $1.5 \mathrm{~mm}$, suspended in articulate form on the supporting beam and joined inseparably with the rapper rod (Fig. 1), different in the way of making bolt joints of HUCKBOLT C50L type (Fig. 2).

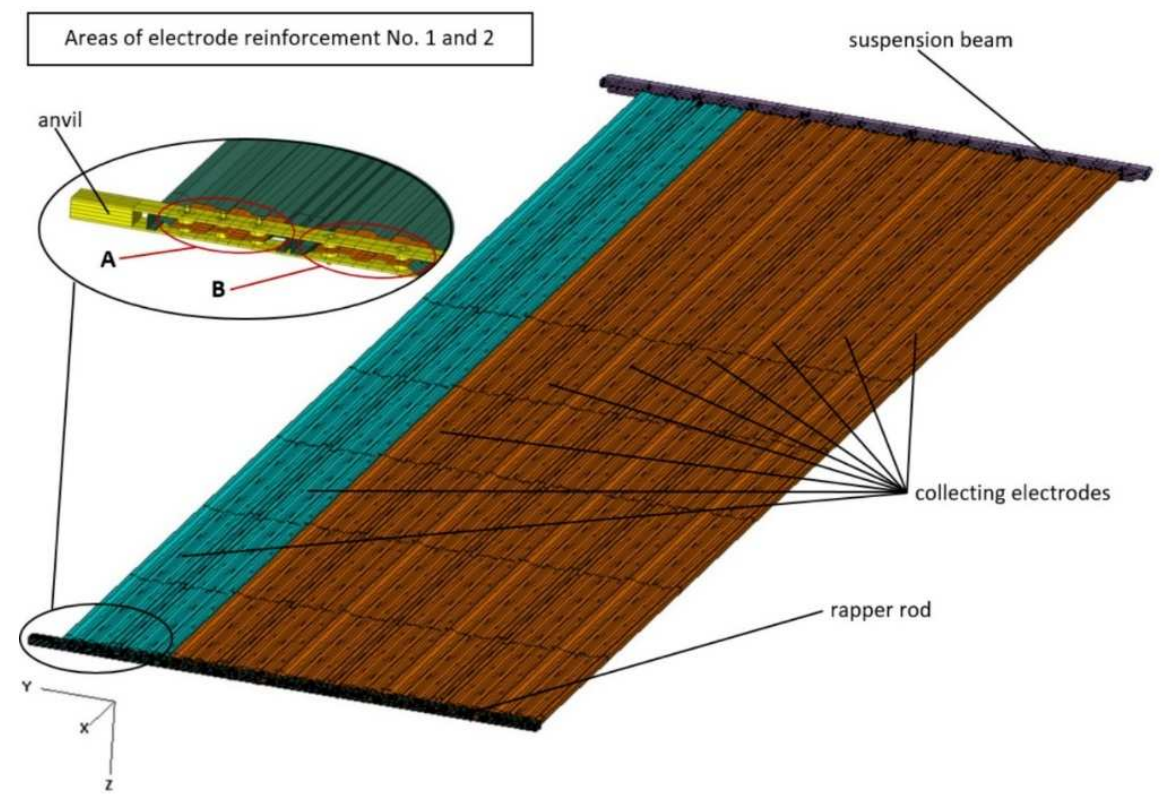

Fig. 1. Diagram of the system of collecting electrodes

\subsection{HUCK type connection models}

For the system of figure 1 two variants of bolt joints were worked out. In the first variant (v.1) the correct geometry of the holes in the rapper rod was modelled (Fig. 2a), i.e. longitudinal axes of the holes in the flat bars of the rapper rod overlap. The other variant (v.2) maps the skew position of the bolt and ring in relation to the holes in the rod - the axes of the bolt and ring are tilted by about $1^{\circ} 30^{\prime}$ from the longitudinal axes of the holes in the flat bars "a" and "b" (Fig. 2b). This is the consequence of the displacement of the axis of holes by $3 \mathrm{~mm}$ in the flat bar "a" in relation to the relevant axes of holes in the flat bar "b" in the plane $\mathbf{x z}$ (system of coordinates $\mathbf{x y z}$, as in figures 1 and 3), in the towards axis $\mathbf{x}$. 
a)

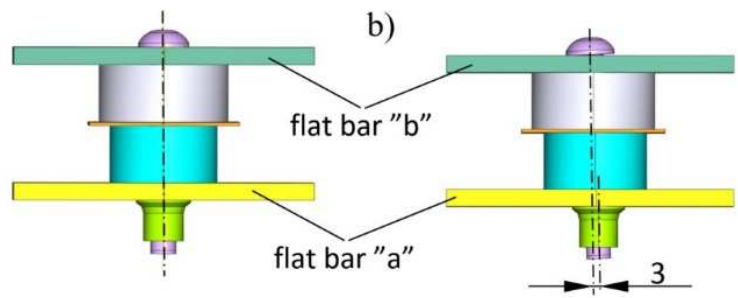

Fig. 2. Variants of bolt joints: a) v.1 - joint technologically correct, b) v.2 - skew joint

\section{Calculations}

As mentioned in the introduction, discrete models of the collecting electrode system and bolt joints were used in numeric calculations to designate stress reduced in bolt joints, paying particular attention to the zone of the contact of the bolt with the ring. The method of numbering bolts and the coordinate system $\mathbf{X Y Z}$ was accepted as shown in Figure 3.

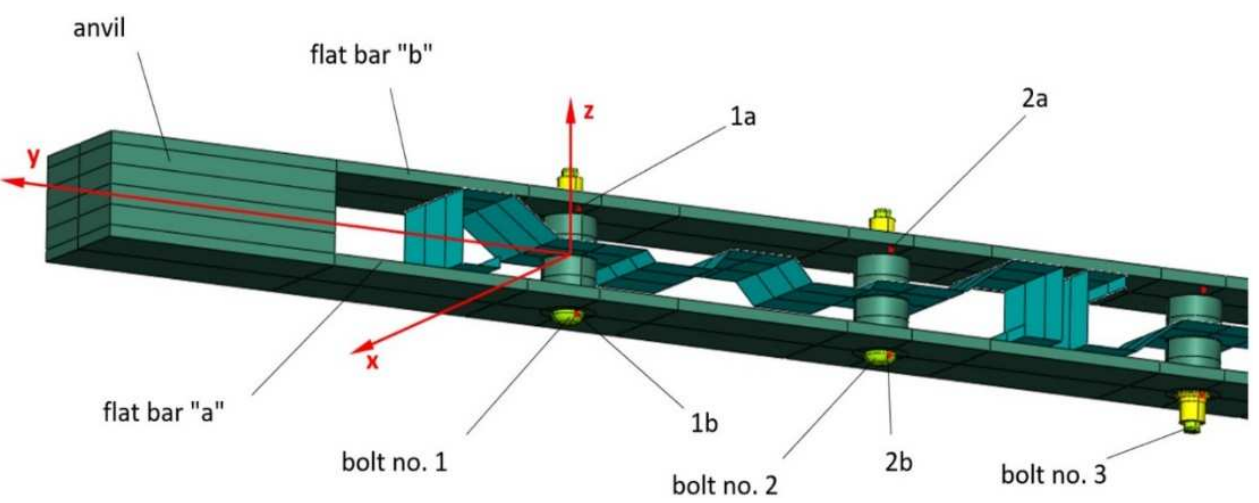

Fig. 3. The numbering of bolt joints and check points of the rapper rod

In both variants the system modelled was loaded with the impulse force corresponding to the impact of a hammer with a weight of $8 \mathrm{~kg}$ against the anvil, with the run obtained from measurements on the test bench. In addition, in bolt joints the force of pre-stress equal to $N=53.5 \mathrm{kN}$ or $N_{\text {nom }}=62 \mathrm{kN}$ was taken into account. The time of the analysis in each of the variants was $0.01 \mathrm{~s}$. Calculations were made with a variable integration step, and the results were recorded in each iteration. In the calculations shown in the further part of study the following physical and geometric parameters were accepted: steel with density $7850 \mathrm{kgm}^{-3}$, Young's modulus $E=206 \mathrm{GPa}$, Poisson's ratio $v=0.3$, coefficient of friction in joints $\mu=0.4$.

\subsection{Acceleration in the rapper rod}

Acceleration in the rapper rod induced by the impulse of impact was determined in the model for variant v. 1. As a result of the simulation performed, runs of acceleration at check points of the rod were obtained. For these runs the maximum values of the constituents of total acceleration were determined (Fig. 4). 


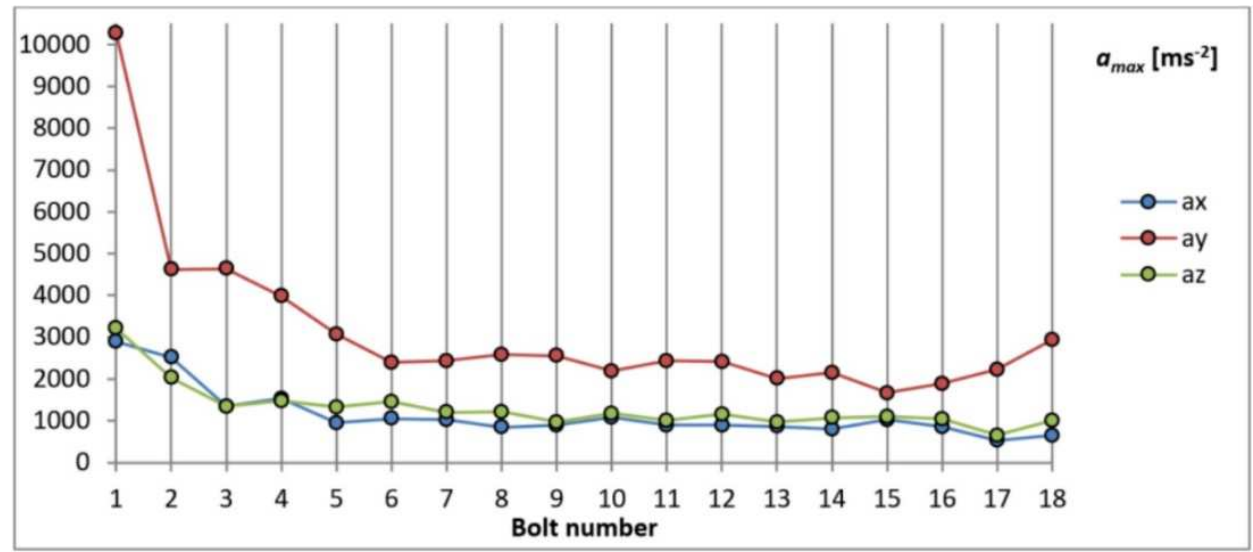

Fig. 4. The maximum total acceleration at checkpoints of the rod (variant v.1)

The analysis of these values shows that the greatest acceleration is at the points closest to the anvil, which is also in line with the experimental data and is one of the elements of model validation [1], [6].

\subsection{Stress analysis in the bolts}

In Figures 5 and 6 the distribution of reduced stresses $\sigma_{\text {red }}$ typical for the variants analysed are shown, found in the elements being part of the joint (fragments of flat bars of the rod, EZ fragment, spacer sleeves, bolt and ring of the bolt). The status of stresses after loading the joint with only minimal force of pre-stress- $N_{\min }=53.5 \mathrm{kN}$ is shown on them. And since in the analysed joint tensile stresses are predominant, according to the Mises hypothesis there was reference to the condition: $\sigma_{l} \cong \sigma_{n} \leq \sigma_{\text {lim }}$.
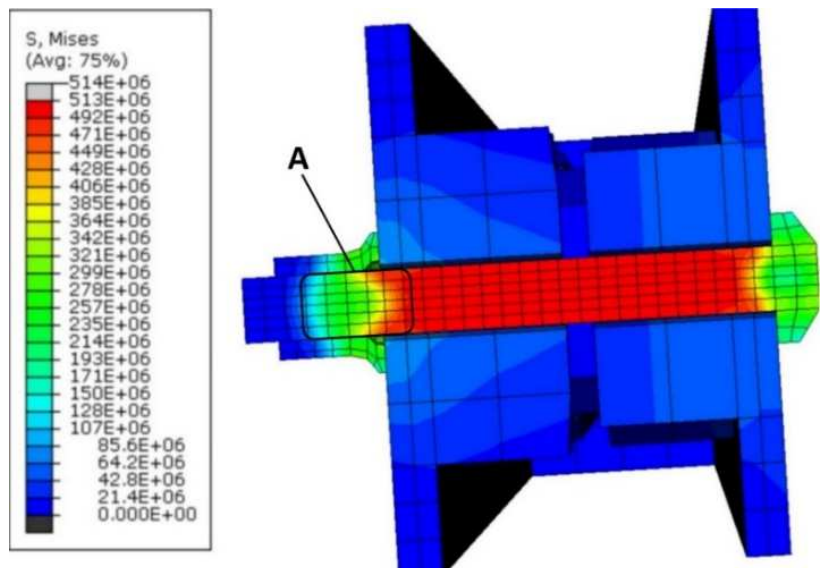

Fig. 5. Distribution of stresses reduced in the bolt joint of joint No 1 under the action of force of prestress equal to $53.5 \mathrm{kN}$ (variant. 1)

Area A (Fig. 5) constitutes a sensitive zone of the bolt because of the largest stresses this is the area of necking in the real bolt, i.e. change of the diameter of the bolt and the area where grooves appear at the entrance to the clamping ring. It should be noted that the A area includes the same zone of the bolt where in the actual objects its cracking was found. This fact confirms the correctness of the calculation model accepted and its conformity with the real object. 
The model of the bolt joint in variant 2 mapped the skew position of the bolt and ring relative to the holes in the rod. As a result of shifting the axis of holes in the flat bars of the rod in areas B, C, D concentrations of stresses appear induced by the non-concentric arrangement of the ring relative to the bolt during pressure (areas B and C). The cause of stresses in region D is, inter alia, the lack of adherence of the frontal area of the head of the bolt to the side area of the rod (E).

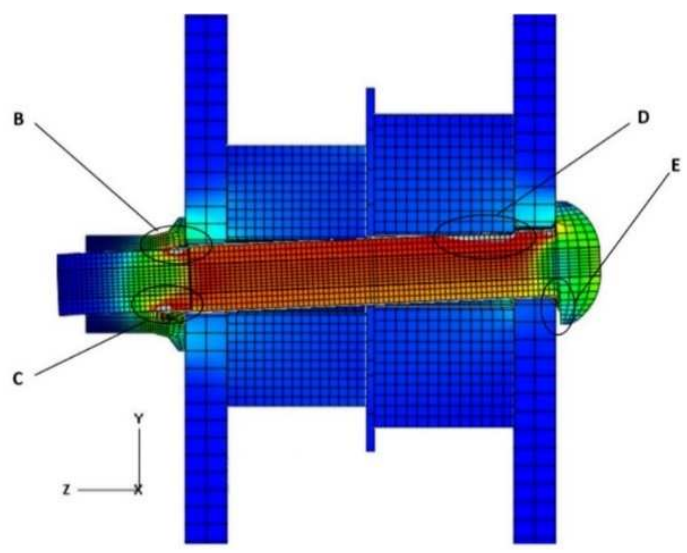

Fig. 6. Distribution of stresses reduced in the bolt joint of joint No 1 under the action of force of prestress equal to $53.5 \mathrm{kN}$ (variant. 1)

Under the load of pre-stress (from the minimum stress force of the rivet) during clenching, the maximum value of reduced stresses does not exceed the variations in v. 1 and v.2, in any bolts respectively: $530 \mathrm{MPa}$, which is approx. $76 \%$ of the permissible tensile stress, which is $\sigma_{\text {lim }}=699 \mathrm{MPa}$ and $602 \mathrm{MPA}$ for the cross-section of the bolt tested, i.e. approx. $86 \%$ of the permissible tensile stress $\sigma_{\text {lim }}$. The value of the maximum permissible tensile stress $\sigma_{\text {lim }}$ was determined taking into account the minimum diameter of the bolt $d_{s, \min }=(11.9 \pm 0.15 \mathrm{~mm})=11.75 \mathrm{~mm}$ appearing in area A, and the acceptance of permissible tensile strength, which according to the manufacturer of the bolts is $N_{\max }=75.8 \mathrm{kN}$.

In the case of the impulse force, reduced stresses occurring in the system are variable in time. In particular, in the bolts they oscillate around the "fixed" value coming from the force of pre-stress - figure 7 shows, for example, oscillations of reduced stress values in the selected finite element in area A of bolt Nos. 1, 5, 14 and 18 for variant v. 1. The oscillations of the values of reduced stresses in variant v.2. are of the same nature.

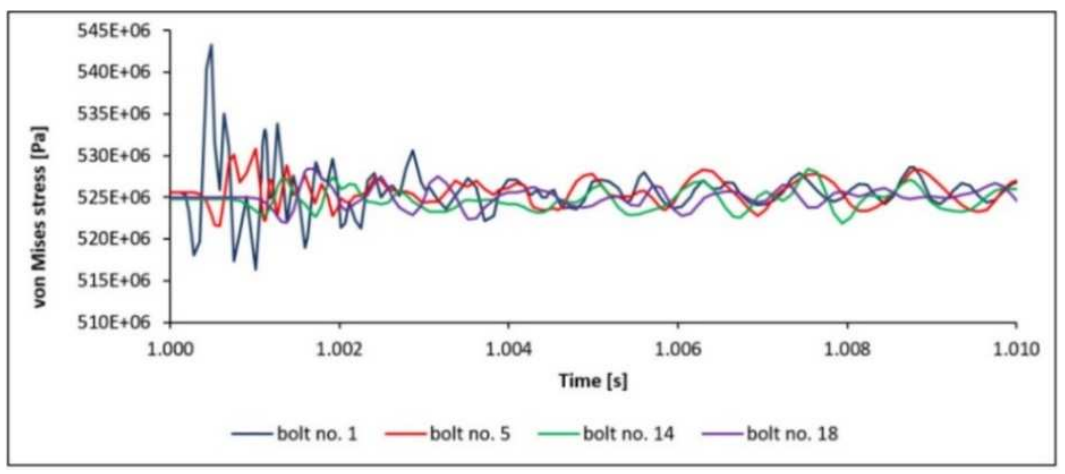

Fig. 7. Oscillations of reduced stress values in one of the finite elements of area A in bolt Nos. 1, 5, 14 and 18 (variant v. 1, the static load of bolts from the pre-stress in combination, and the load of the whole system with impulse force when the hammer hits the anvil) 
Figure 8 shows the comparison of the values of oscillation amplitudes of reduced stresses in all bolt joints in both variants.

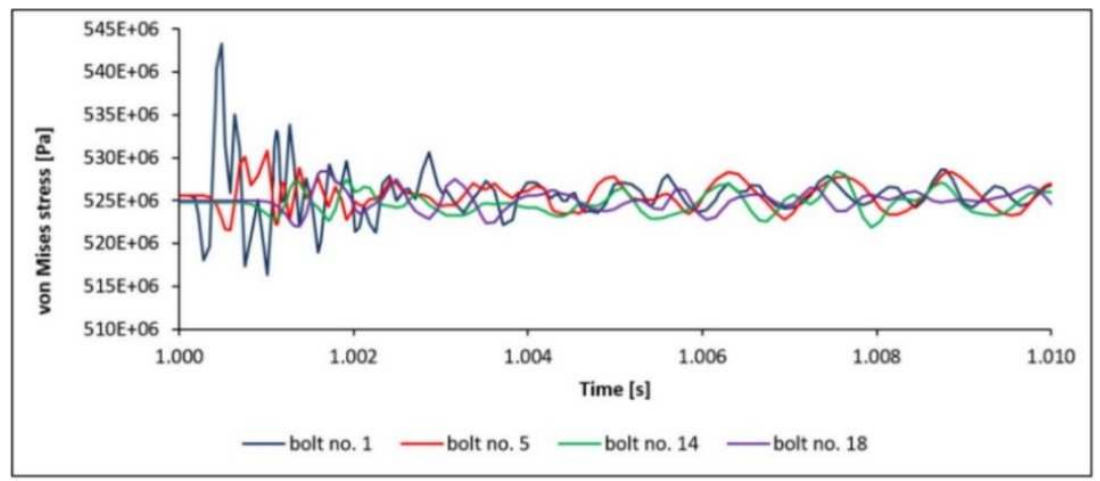

Fig. 8. Amplitudes of reduced stresses in the bolt joint of joint No 1 under the action of force of prestress equal to $53.5 \mathrm{kN}$ (variant. 1 and variant 2)

These analyses were performed for the minimum calculation stress force stressing the bolt $N_{\text {min }}=53.5 \mathrm{kN}$ specified by the manufacturer. According to the manufacturer's data, the actual stressing force can reach in some rivets higher values, of $N_{\text {nom }}=62.0 \mathrm{kN}$. This is due to the technology of clenching the rivet - by breaking the mandrel in the necking, during the operation of its stressing. For this increased force of stressing, on the basis of the assumptions specified earlier, Figure 9 shows the distribution of the maximum reduced stresses in the joints of bolts.

In conclusion, in variant v.1, with the bolts joints made in the right way, the maximum values of total reduced stresses - from the loads of bolt stress and the impulse of the impact of the hammer against the anvil, do not exceed the $634 \mathrm{MPa}$. In each of the bolts they are much lower than the stresses permissible for this type of bolt, which according to the data of the manufacturer are $\sigma_{\text {lim }}=699 \mathrm{MPa}$ and significantly below the average tensile strength of the material of the bolt specified for $R_{m} \approx 851,5 \mathrm{MPa}$. The area of maximum stresses in the bolt is located in region A (Fig. 5), i.e. in the section of clenching the bolt with the sleeve. As can be seen in each of the bolts, stresses are well below the permissible tensile stress. In the case of variant v. 2 in the bolts of joint Nos. 1 and 2 the value $\sigma_{\text {lim }}=699 \mathrm{MPa}$ was exceeded, and in bolt No. 3 the value of reduced stresses received is close to $\sigma_{\text {lim }}$.

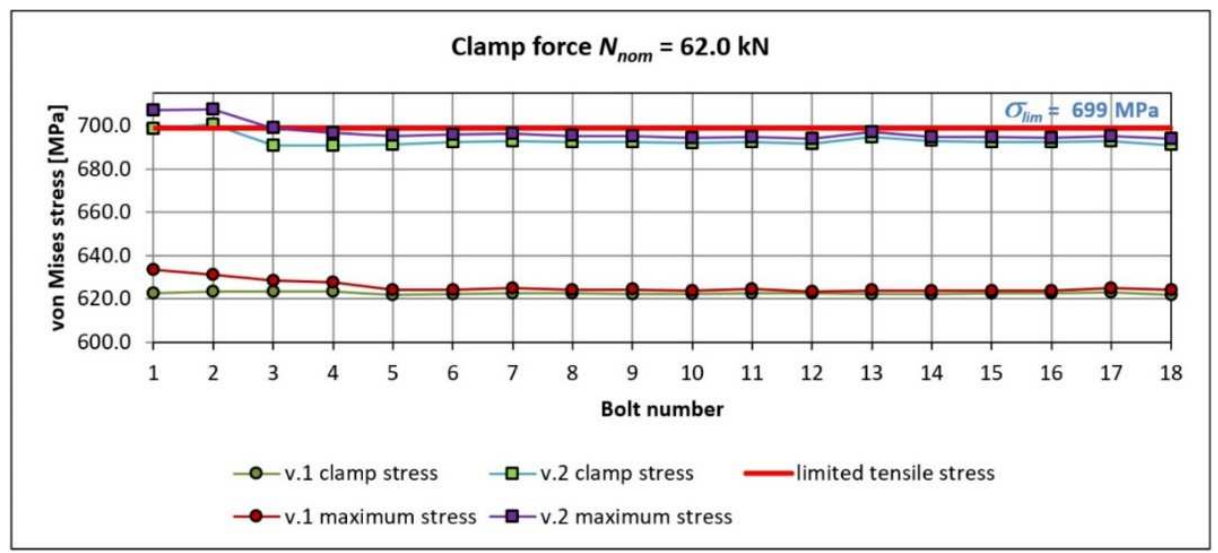

Fig. 9. Distribution of the maximum reduced stresses in the joints of bolt Nos. $1 \div 18$ (variants v. 1 and v.2) under the action of only the pre-load with the stress of force $\mathrm{N}_{\text {nom }}=62.0 \mathrm{kN}$ (green) or preload from pre-stress with force $\mathrm{N}_{\text {nom }}$ and the impulse force of impact (red) 
For the quantitative evaluation of dynamic stresses caused by the impulse force of the impact of the hammer against the anvil, the comparison of the effective values of the runs of these stresses was applied. After isolating the dynamic runs of reduced stresses, the effective values of reduced stresses were determined. It was accepted that the total effective value of reduced stresses in all joints is equal to $100 \%$. The comparison of the relative effective values (i.e. related to the total values) allows, indirectly, the quantitative evaluation of the share of individual bolt joints in the transfer of energy - from the impulse force - from the rod to electrodes. Such a comparison for variant v.1 is shown in Figure 10.

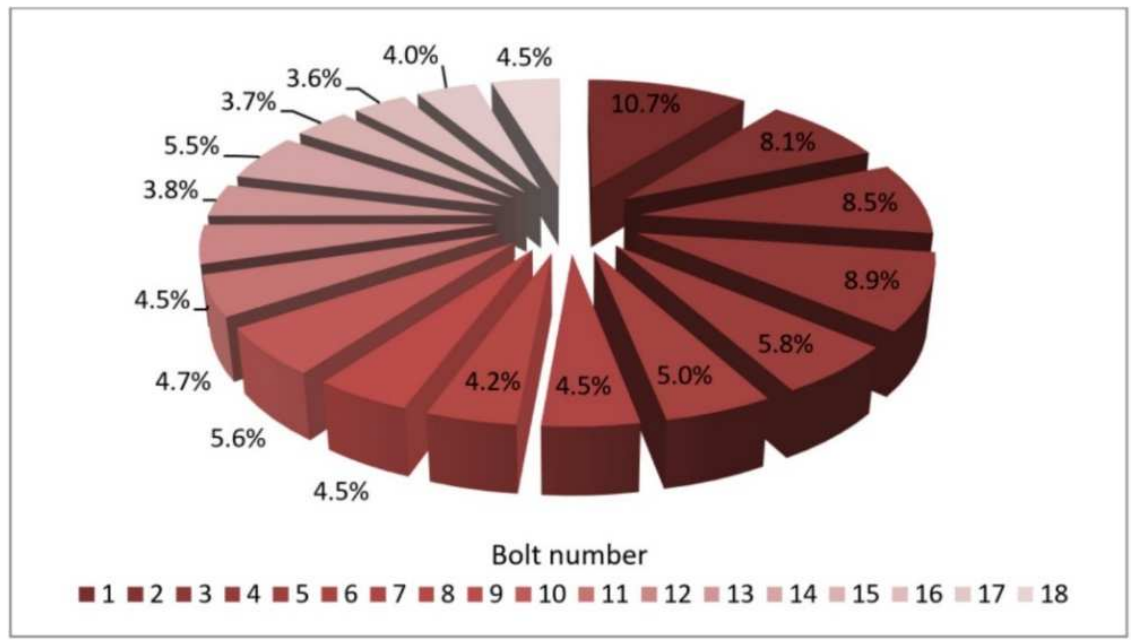

Fig. 10. The share of bolt joints in the transfer of energy from the impact of the hammer against the anvil to electrodes (variant v.1)

It can be noted that the first four joints from the side of the anvil (bolts no. $1 \div 4$ ) transfer more than $36 \%$ of the energy of impact, including bolt joint No 1, almost $11 \%$. This indicates that the most vulnerable to the impact of dynamic loads are bolts in joint Nos. 1 to No 4 from the side of the anvil. In other rivet joints this impact is significantly smaller. In variant v. 2 similar dependencies take place.

\section{Conclusion}

The analyses shown in this study make it possible to conclude that with the bolt joints made properly (variant v.1) the maximum values of total reduced stresses - from the loads of the pre-stress of the bolt and the impulse force of the impact of hammer against the anvil in each of the bolts are significantly lower than the values of permissible stresses. However, the incorrect workshop execution of bolt joints, involving the non-alignment of the pairs of holes on the rivets, results in the skew position of the bolt in the holes during the installation of electrodes. The calculation analysis of such joints (variant v.2) showed a significant increase of pre-stresses in the bolts as a result of additional bending during stress. In special cases of the simultaneous occurrence of the skew execution of the joint and pre-stressing with the maximum force $N_{\text {nom }}$ bolt joints which are on the electrodes close to anvils can be damaged, which in turn will result in the decrease of the effectiveness of the rapping of these electrodes, resulting in a decrease in the efficiency of cleaning exhaust gases in the chamber of the electrostatic precipitator. 


\section{References}

1. Nowak A. Vibration of collecting electrodes in electrostatic precipitators - modelling, measurements and simulation tests, Journal of Electrostatics, 70, 3, pp. 327-332, (2012)

2. Nowak A., Nowak P., Awrejcewicz J. Influence of impulse force loading on vibrations of the collecting electrodes, International Journal of Structural Stability and Dynamics, 17, 5, pp. 1740011-1-1740011-15, (2017)

3. Zienkiewicz O.C., Taylor R.L.: The Finite Element Method, Vol. 2 Solid Mechanics, Fifth ed., Butterworth-Heinemann, (2000)

4. Zienkiewicz O.C., Taylor R.L., Zhu J.Z.: The Finite Element Method, Its Basis\&Fundamentals, Sixth ed., Butterworth-Heinemann, (2005)

5. Rakowski G., Kacprzyk Z.: Metoda elementów skończonych w mechanice konstrukcji, Oficyna Wydawnicza Politechniki Warszawskiej, Warszawa (2005)

6. Nowak A., Nowak P., Kurz M., Ryguła C. Study of the effect of the stiffness of the anvil beam for vibration propagation in the system of collecting electrodes, Przegląd Elektrotechniczny, pp. 232-236, (2017) 